\title{
CARACTERÍSTICAS FÍSICAS Y VALOR NUTRITIVO DEL ENSILAJE DE MORERA (Morus alba) MEZCLADO CON FORRAJE DE MAÍZ1
}

\author{
Carlos Boschini ${ }^{2}$
}

\begin{abstract}
RESUMEN
Características físicas y valor nutritivo del ensilaje de morera (Morus alba) mezclado con forraje de maíz. Follaje de morera y maíz cosechados a 120 y 135 días respectivamente fueron ensilados, picados y mezclados en proporciones porcentuales de 100:0, 75:25, 50:50, 25:75 y 0:100 en base a la masa seca. La concentración de carbohidratos solubles fue uniformada a $10 \%$ en todos los tratamientos con la adición de melaza en proporción a las mezclas experimentales. El periodo de fermentación fue de 90 días. El forraje de morera (proporción 100:0) compactado tuvo una densidad de $693 \mathrm{~kg} / \mathrm{m}^{3}$ y el forraje de maíz (proporción 0:100) fue de 541 $\mathrm{kg} / \mathrm{m}^{3}$, observándose marcadas diferencias iniciales $(\mathrm{P} \leq 0,01)$ entre las mezclas forrajeras. Al final del periodo experimental, el ensilado mostró densidades entre 750 y $556 \mathrm{~kg} / \mathrm{m}^{3}$, con valores intermedios $(\mathrm{P} \leq 0,01)$ al disminuir la proporción de morera en la mezcla. Se detectaron pérdidas de materia seca entre los tratamientos $(\mathrm{P} \leq 0,01)$, entre $\left.6,8 \mathrm{~kg} / \mathrm{m}^{3}\right)$ y 18,3 $\mathrm{kg} / \mathrm{m}^{3}$. La evaluación organoléptica tuvo una calificación de muy bueno a excelente en las mezclas 75:50, 50:50, 25:75 y 0:100, no así en el ensilaje de solo morera (100:0), el cual presentó un ligero olor amoniacal. La concentración de materia seca en el material fresco fue superior al encontrado en el material ensilado $(\mathrm{P} \leq 0,05)$. La concentración de proteína cruda entre las mezclas se observó claramente diferenciada $(\mathrm{P} \leq 0,01)$, tanto al inicio como al final de periodo de fermentación. Similar respuesta se observó en la concentración de fibra neutro detergente y de las cenizas totales.
\end{abstract}

\begin{abstract}
Physical characteristics and nutritional value of stored mulberry (Morus alba) mixed with corn forage. Mulberry foliage and corn, harvested respectively after 120 and 135 days, were ensilaged (chopped and mixed in ratios of 100:0, 75:25, 50:50, 25:75 and 0:100 as dry material). The concentration of soluble carbohydrates was uniform at $10 \%$ in all the treatments by adding molasses. The fermentation period was 90 days. The compacted mulberry forage (the 100:0 ratio) had a density of $693 \mathrm{~kg} / \mathrm{m}^{3}$ and that of the corn forage (the 0:100 ratio) was $541 \mathrm{~kg} / \mathrm{m}^{3}$, indicating marked initial differences $(\mathrm{p} \leq 0.01)$ between them. At the end of the experimental period, the stored material had densities ranging between 750 and $556 \mathrm{~kg} / \mathrm{m}^{3}$, with intermediate values $(p \leq 0.01)$ as the mulberry proportion was reduced in each mixture. Loss of dry material was found among treatments $(\mathrm{p} \leq 0.01)$ with $6.8 \mathrm{~kg} / \mathrm{m}^{3}$ and $18.3 \mathrm{~kg} / \mathrm{m}^{3}$. The organoleptic characteristics evaluation had ratings from very good to excellent in the mixtures with ratios of 75:50, 50:50, 25:75 and 0:100, but not in the mulberry forage (100:0) which has a slight ammonia odor. The concentration of dry matter in the fresh material was higher than that found in the stored material $(\mathrm{p} \leq 0.05)$. The concentration of crude protein among the mixtures was clearly differentiated $(\mathrm{p} \leq 0.01)$, in the beginning as well as at the end of the fermentation period. A similar condition was observed in the concentration of neutral detergent fiber and total ash.
\end{abstract}

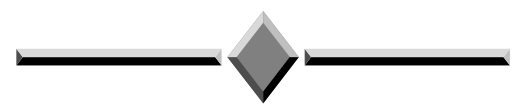

\section{INTRODUCCIÓN}

El ensilaje de maíz ha sido y parece ser el mejor ingrediente forrajero en las raciones para el ganado lechero (Kuehn et al. 1999). Muchas diferencias en el contenido de materia seca y en la digestibilidad del en- silaje de maíz fueron relacionadas con la madurez de la planta y las diferencias entre híbridos seleccionados para la siembra. En este sentido, varios estudios han identificado diferencias importantes en la producción de forraje de la planta entera (Hunt et al. 1993, Roth 1994) y en la composición botánica, como el porcentaje de

\footnotetext{
1 Recibido para publicación el 23 de agosto del 2002. Inscrito en la Vicerrectoría de Investigación, Proyecto No. 737-97-006.

2 Estación Experimental Alfredo Volio Mata. Facultad de Agronomía. Universidad de Costa Rica. E-mail: boschini@cariari.ucr.ac.cr.
} 
elotes (grano) (Hunt et al. 1992). Los análisis de laboratorio han mostrado que las variedades e híbridos de maíz difieren en proteína cruda, en el contenido de fibras neutro (FND) y ácido detergentes (FAD), y en la digestibilidad in vitro (Hunt et al. 1993, Verbic et al. 1995, Leask et al. 1973).

En cultivo para la producción de forraje, la morera ha mostrado excelentes características organolépticas y un alto consumo por el ganado (Benavides et al. 1994, Ortiz 1992, Castro 1989). El contenido proteico de la planta entera varía de 14 a 26\% de proteína cruda en base seca (Boschini et al. 1999) y la digestibilidad in vitro va de 70 a $80 \%$ (Ortiz 1992). La degradabilidad potencial de la hoja va de 86 a $90 \%$, con contenidos de fibra neutro detergente entre 34 y $37 \%$ y de fibra ácido detergente entre 26 y $29 \%$, en las edades de 56 a 112 días de rebrote, con degradabilidades ruminales efectivas de 67 a $78 \%$ a una tasa de pasaje de 4\%/h (Boschini 2001). Muestras de morera analizadas por UFAG Laboratorien de Suiza, provenientes de la zona alta de Cartago en la Meseta Central de Costa Rica, presentaron valores en base seca de $22 \%$ de proteína cruda, $19 \%$ de fibra cruda, $2,3 \%$ de extracto etéreo, $50 \%$ de proteína sobrepasante y una estimación de 1,48 Mcal/kg de energía neta para lactación. Eswara et al. 1992, evaluando el valor nutritivo de la hojas de morera, han reportado consumos de materia seca de 2,74 a $3,55 \%$ del peso vivo en cabras y ovejas, con contenidos de $12 \%$ de proteína cruda y $71 \%$ de TND.

Ambas fuentes forrajeras, la morera con un alto contenido de proteína y el maíz con una alta concentración de energía y fuente de fibra de buena calidad, parecen constituir una combinación forrajera ideal para la alimentación de vacas lecheras. El cultivo de ambas especies, en igualdad altitudinal, muestran un comportamiento fenológico similar, para obtener rendimientos y estados de aprovechamiento óptimos como alimento forrajero (Boschini 1998, 1999, Amador et al. 2000, Elizondo et al. 2002). La cosecha de forraje en lotes obliga a la conservación de ellos, mediante el ensilaje para aprovecharse posteriormente en porciones, distribuidas a lo largo de un periodo preestablecido a gusto del ganadero. El presente trabajo se llevó a cabo con el propósito de conocer la combinación de diferentes proporciones de morera y maíz en la calidad bromatológica del ensilado.

\section{MATERIALES Y MÉTODOS}

El experimento se llevó a cabo en la Estación Experimental de Ganado Lechero "Alfredo Volio Mata" de la Universidad de Costa Rica durante el año 2001. La morera se extrajo de una plantación con seis años de establecida, siendo uniformada a una altura de $60 \mathrm{~cm}$ sobre el nivel del suelo. Dos semanas después fue fertilizada con nitrato de amonio con un equivalente de $150 \mathrm{~kg} / \mathrm{ha} / \mathrm{año}$ de $\mathrm{N}_{2}$. Una parcela de maíz criollo fue sembrada, con una distancia entre surcos de $70 \mathrm{~cm}$ y una distancia entre plantas de $50 \mathrm{~cm}$, con tres semillas en cada hoyo de siembra. El cultivo fue fertilizado previo a la siembra con $250 \mathrm{~kg} / \mathrm{ha}$ de la fórmula 10-30-10 $\left(\mathrm{N}_{2}, \mathrm{P}_{2} \mathrm{O}_{4}, \mathrm{~K}_{2} \mathrm{O}\right)$ y a los 42 días posteriores a la siembra con $46 \mathrm{~kg} / \mathrm{ha}$ con nitrato de amonio.

La biomasa de morera (hojas y tallos) se cosechó a los 120 días de rebrote y la de maíz a los 135 días de edad. Ambos materiales fueron picados, por separado, con un tamaño de partícula de $20 \mathrm{~mm}$. Posteriormente, se prepararon las mezclas experimentales de morera y maíz en las proporciones porcentuales de 100:0, 75:25, 50:50, 25:75 y 0:100 en base a la masa seca y se extrajeron muestras para determinar la composición bromatológica . Cada mezcla experimental fue vertida en silos metálicos con capacidad de 200 litros, con una llave en el fondo para obtener efuentes, efectuándose la compactación en forma manual con un apizonador de madera de $10 \mathrm{~cm}$ de diámetro. Cada tratamiento experimental tuvo cuatro repeticiones. La concentración de carbohidratos solubles en la morera se estimaron en $4 \%$ y del maíz en $8 \%$ en base seca. Con el propósito de uniformar la concentración de carbohidratos solubles a $10 \%$ en todos los tratamientos, se adicionó melaza (35\% CHOS solubles) en la proporción correspondiente a las diferentes mezclas experimentales de morera y maíz. Cada silo llenado fue cubierto con un plástico negro dejando un sobrante de $20 \mathrm{~cm}$ a su alrededor y sobre él se colocó una tapa metálica con un aro de amarre hermético. Los silos metálicos fueron pesados antes y después de llenados. Durante las primeras cuatro semanas la llave de efluentes fue abierta para recoger la fracción líquida y posteriormente cada dos semanas.

El periodo experimental fue de 90 días. Al cabo de este periodo, los silos fueron abiertos. Se pesaron nuevamente y se taró su contenido con base al peso inicial de los envases, también fue medida la pérdida de volumen de la masa ensilada. Se realizó una evaluación organoléptica mediante la conformación de un panel de tres expertos y se extrajo una muestra compuesta para los análisis químicos. En el laboratorio se determinó la concentración de materia seca, proteína cruda y cenizas totales mediante los procedimientos aprobados por la AOAC (1980), así como la fibra neutro detergente, fibra detergente ácida, hemicelulosa, lignina y celulosa con los métodos descritos por Gering y Van Soest 
(1970), tanto el material inicial como del obtenido al final del proceso de fermentación. Los resultados fueron sometidos al análisis de variación con un modelo de dos caminos de clasificación: una fuente con cinco tratamientos y una fuente con cuatro repeticiones. Se efectuó la separación de medias mediante la prueba de Duncan. Los datos fueron analizados empleando el PROC REG del paquete estadístico SAS (1985).

\section{RESULTADOS}

La cantidad de materia fresca empleada en el llenado de los silos fue de 103,5 a 145,3 kg/silo. Esta variación se debió a las diferentes densidades de las mezclas de morera y maíz experimentadas. El Cuadro 1 muestra que el forraje fresco de morera (proporción 100:0) compactado tuvo una densidad inicial de $693 \mathrm{~kg} / \mathrm{m}^{3} \mathrm{y}$ con el forraje fresco de maíz (proporción 0:100) compactado fue de $541 \mathrm{~kg} / \mathrm{m}^{3}$, observándose marcadas diferencias iniciales $(\mathrm{P}<0,01)$ entre las diferentes mezclas forrajeras. Al final del periodo experimental, el forraje ensilado mostró densidades entre 750 y $556 \mathrm{~kg} / \mathrm{m}^{3}$, con valores intermedios $(\mathrm{P}<0,01)$ al disminuir la proporción de morera en la mezcla. La pérdida de volumen entre el inicio y el final del experimento varió de 27,9 a 86,7 $1 / \mathrm{m}^{3}$, siendo de un $12,5 \%$ para la mezcla 100:0 (solo morera), de $10,1 \%$ para la mezcla $0: 100 \%$ (solo maíz) y de un 5\% para las mezclas 75:25, 50:50 y 25:75. Se detectaron pérdidas de materia seca entre los tratamientos $(\mathrm{P}<0,01)$, entre $6,8 \mathrm{~kg} / \mathrm{m}^{3}$ en el tratamiento $0: 100$ (solo maíz) y $18,3 \mathrm{~kg} / \mathrm{m}^{3}$ en la mezcla $75: 25$.

La evaluación organoléptica mostró uniformidad de criterios entre los expertos, con una calificación de muy bueno a excelente en las mezclas 75:50, 50:50, 25:75 y 0:100. El ensilaje de solo morera (100:0), pre- sentó una excelente apariencia y coloración, sin embargo se detectó un ligero olor amoniacal.

En el Cuadro 2 se presenta la composición química del forraje ensilado al inicio y al final del experimento. La concentración de materia seca en el material fresco fue superior al encontrado en el material ensilado $(\mathrm{P}<0,05)$, así como la concentración de proteína cruda en las mezclas 100:0 y 75:25. Al aumentar la proporción de maíz en las mezclas, la concentración de proteína en la materia seca del ensilado fue ligeramente superior al hallado en la materia seca de la mezcla inicial. La concentración de proteína cruda entre las mezclas se observa claramente diferenciada $(\mathrm{P}<0,01)$, tanto al inicio como al final de periodo de fermentación. Similar respuesta se observó en la concentración de fibra neutro detergente y de las cenizas totales. La concentración de fibra neutro detergente es en general ligeramente superior en la materia seca inicial. Sin embargo, durante el proceso de fermentación, la concentración de las fracciones de la fibra detergente presentaron también una pequeña variación, se observó un ligero aumento en la concentración de fibra detergente ácida y una disminución en la concentración de hemicelulosa. Entre las fracciones de la fibra ácida, las concentraciones de celulosa y lignina al final del proceso de fermentación fueron mayores que las determinadas en el forraje inicial. En el Cuadro 3 se presenta la cantidad de materia seca y de nutrientes por unidad de masa al inicio y al final del periodo experimental en las diferentes mezclas probadas. La cantidad de materia seca de las mezclas experimentales, puesta inicialmente fue entre 210 y $224 \mathrm{~kg} / \mathrm{t}$ de materia verde, observándose al final del experimento cantidades de materia seca entre 177 y $192 \mathrm{~kg} / \mathrm{t}$ entre los tratamientos. La pérdida de materia seca ocurrida durante el proceso de fermentación fue de $14 \%$ en la mezcla 100:0, disminuyendo progresivamente a $11,4 \%$ en la mezcla $25: 75$ conforme

Cuadro 1. Densidad del material ensilado al inicio y al final del periodo experimental. Ochomogo, Cartago, Costa Rica. 2001.

\begin{tabular}{|c|c|c|c|c|c|c|c|c|}
\hline \multirow{2}{*}{\multicolumn{3}{|c|}{$\begin{array}{c}\text { Proporción del } \\
\text { material ensilado }\end{array}$}} & \multicolumn{2}{|c|}{$\begin{array}{c}\text { Densidad } \\
\text { en base fresca }\end{array}$} & \multirow{3}{*}{$\begin{array}{c}\text { Pérdida } \\
\text { de volumen } \\
1 / \mathbf{m}^{3}\end{array}$} & \multicolumn{2}{|c|}{$\begin{array}{c}\text { Densidad } \\
\text { en base fresca }\end{array}$} & \multirow{3}{*}{$\begin{array}{r}\text { Pérdida } \\
\text { del MS } \\
\mathrm{kg} / \mathrm{m}^{3}\end{array}$} \\
\hline & & & \multirow{2}{*}{$\begin{array}{l}\text { inicial } \\
\mathrm{kg} / \mathrm{m}^{3}\end{array}$} & \multirow{2}{*}{$\begin{array}{c}\text { final } \\
\mathrm{kg} / \mathrm{m}^{3}\end{array}$} & & \multirow{2}{*}{$\begin{array}{r}\text { inicial } \\
\mathrm{kg} / \mathrm{m}^{3}\end{array}$} & \multirow{2}{*}{$\begin{array}{c}\text { final } \\
\mathrm{kg} / \mathrm{m}^{3}\end{array}$} & \\
\hline Morera & : & Maíz & & & & & & \\
\hline 100 & : & 0 & $693 \mathrm{a}$ & $750 \mathrm{a}$ & 86,7 a & $155,8 \mathrm{a}$ & $144,5 \mathrm{a}$ & $11,3 \mathrm{c}$ \\
\hline 75 & : & 25 & $677 \mathrm{a}$ & $684 \mathrm{~b}$ & $35,2 \mathrm{c}$ & $149,5 \mathrm{a}$ & $131,3 \mathrm{~b}$ & $18,3 \mathrm{a}$ \\
\hline 50 & : & 50 & $633 \mathrm{~b}$ & $633 c$ & $34,9 \mathrm{c}$ & $137,5 \mathrm{~b}$ & $119,8 \mathrm{c}$ & $17,8 \mathrm{a}$ \\
\hline 25 & : & 75 & $598 \mathrm{c}$ & $600 \mathrm{~d}$ & $27,9 \mathrm{~d}$ & $128,3 \mathrm{c}$ & $114,3 \mathrm{~cd}$ & $14,0 \mathrm{~b}$ \\
\hline 0 & : & 100 & $541 \mathrm{~d}$ & $556 \mathrm{e}$ & $54,8 \mathrm{~b}$ & $115,0 \mathrm{~d}$ & $108,3 \mathrm{~d}$ & $6,8 \mathrm{~d}$ \\
\hline
\end{tabular}

a, b, c, d, e marca las diferencias significativas $(\mathrm{P}<0,05)$ entre tratamientos. 
Cuadro 2. Composición química del material ensilado al inicio y al final del experimento. Ochomogo, Cartago, Costa Rica. 2001.

\begin{tabular}{|c|c|c|c|c|c|}
\hline & \multicolumn{5}{|c|}{ Proporción de morera:maíz en el material ensilado } \\
\hline & 100:0\% & $75: 25 \%$ & $\mathbf{5 0 : 5 0 \%}$ & $25: 75 \%$ & $0: 100 \%$ \\
\hline \multicolumn{6}{|l|}{ Materia seca (\%) } \\
\hline Inicial & 22,44 a & $22,10 \mathrm{~b}$ & $21,75 \mathrm{c}$ & $21,40 \mathrm{~d}$ & $21,04 \mathrm{e}$ \\
\hline Final & $19,28 \mathrm{a}$ & 19,16 a & 19,03 a & $18,95 \mathrm{a}$ & $17,72 \mathrm{~b}$ \\
\hline \multicolumn{6}{|l|}{ Proteina cruda $(\%)$} \\
\hline Inicial & $16,15 \mathrm{a}$ & $14,07 \mathrm{~b}$ & $11,96 \mathrm{c}$ & $9,83 \mathrm{~d}$ & $7,68 \mathrm{e}$ \\
\hline Final & 13,91 a & 13,86 a & $12,85 \mathrm{~b}$ & $10,51 \mathrm{c}$ & $8,41 \mathrm{~d}$ \\
\hline \multicolumn{6}{|l|}{ FND $(\%)$} \\
\hline Inicial & $49,70 \mathrm{e}$ & $54,23 \mathrm{~d}$ & $58,81 \mathrm{c}$ & $63,44 \mathrm{~b}$ & 68,12 a \\
\hline Final & $50,37 \mathrm{~b}$ & $51,04 \mathrm{~b}$ & $53,74 \mathrm{~b}$ & $60,84 \mathrm{a}$ & 64,69 a \\
\hline \multicolumn{6}{|l|}{ FAD $(\%)$} \\
\hline Inicial & $32,92 \mathrm{e}$ & $35,42 \mathrm{~d}$ & $37,94 \mathrm{c}$ & $40,49 \mathrm{~b}$ & $43,07 \mathrm{a}$ \\
\hline Final & $36,48 \mathrm{~b}$ & $39,39 \mathrm{ab}$ & 40,31 a & $41,89 \mathrm{a}$ & $42,51 \mathrm{a}$ \\
\hline \multicolumn{6}{|l|}{ Hemicelulosa (\%) } \\
\hline Inicial & $16,78 \mathrm{e}$ & $18,81 \mathrm{~d}$ & $20,87 \mathrm{c}$ & $22,95 \mathrm{~b}$ & $25,05 \mathrm{a}$ \\
\hline Final & $13,89 \mathrm{~b}$ & $11,65 \mathrm{~b}$ & $13,43 \mathrm{~b}$ & $18,95 \mathrm{a}$ & $22,18 \mathrm{a}$ \\
\hline \multicolumn{6}{|l|}{ Lignina $(\%)$} \\
\hline Inicial & $6,25 \mathrm{e}$ & $6,28 \mathrm{~d}$ & $6,30 \mathrm{c}$ & $6,33 \mathrm{~b}$ & $6,36 \mathrm{a}$ \\
\hline Final & $6,85 \mathrm{a}$ & $7,04 \mathrm{a}$ & $7,09 \mathrm{a}$ & $6,64 \mathrm{a}$ & $5,81 \mathrm{~b}$ \\
\hline \multicolumn{6}{|l|}{ Celulosa (\%) } \\
\hline Inicial & $26,67 \mathrm{e}$ & $29,14 \mathrm{~d}$ & $31,64 \mathrm{c}$ & $34,16 \mathrm{~b}$ & $36,71 \mathrm{a}$ \\
\hline Final & $29,63 \mathrm{c}$ & $32,35 \mathrm{c}$ & $33,22 \mathrm{bc}$ & $35,25 \mathrm{ab}$ & $36,70 \mathrm{a}$ \\
\hline \multicolumn{6}{|l|}{ Cenizas (\%) } \\
\hline Inicial & $14,95 \mathrm{a}$ & $13,03 \mathrm{~b}$ & $11,08 \mathrm{c}$ & $10,40 \mathrm{~d}$ & $9,10 \mathrm{e}$ \\
\hline Final & $14,44 \mathrm{a}$ & $13,72 \mathrm{a}$ & $12,27 \mathrm{~b}$ & $10,89 \mathrm{c}$ & $8,79 \mathrm{~d}$ \\
\hline
\end{tabular}

a, b, c, d, e marca las diferencias significativas $(P<0,05)$ entre tratamientos.

aumentó la proporción de maíz en las mezclas. Esta pérdida de materia seca aumentó a 15,8\% en la concentración 0:100 (solo maíz). La cantidad de proteína cruda en la materia verde inicial varió de 36,2 a $16,2 \mathrm{~kg} / \mathrm{t}$ de materia verde entre las mezclas de 100:0 y 0:100. Al final del periodo experimental, la cantidad de proteína cruda encontrada fue entre 26,8 y $14,9 \mathrm{~kg} / \mathrm{t}$ de materia verde. La pérdida de proteína cruda $(\mathrm{P}<0,01)$ fue alta (14-26\%) en las mezclas con mayor proporción de morera (100:0 y 75:25), representando entre 2 y $4 \%$ de la materia seca, y baja (5-7\%) en las mezclas de 50:50, $25: 75$ y $0: 100$, entre 0,5 y $0,7 \%$ de la materia seca. Las pérdidas de fibra neutro detergente fueron entre 14,4 en la mezcla $100: 0$ y $28,7 \mathrm{~kg} / \mathrm{t}$ de materia verde en la mezcla de $0: 100$, con valores proporcionales a la composición de materiales en las restantes mezclas, si se miden en relación porcentual a la cantidad de materia seca inicial. Similar respuesta se observa en las fracciones de la fibra detergente.

\section{DISCUSIÓN}

La morera fresca recién ensilada (mezcla 100:0) mostró una densidad de $152 \mathrm{~kg} / \mathrm{m}^{3}$ (28\%) mayor que en el maíz (mezcla 0:100). Teóricamente se espera que esa diferencia, partiendo de la mezcla con solo maíz, incremente la densidad en un $7 \%$ con cada adición de $25 \%$ de morera en la mezcla fresca, Sin embargo, los resultados muestran que en la mezcla 25:75 la densidad incrementó $10.5 \%$, en la mezcla $50: 50$ un $17 \%$ y en la mezcla 75:25 fue de 25,1\%. Al final del periodo de fermentación, los materiales puros de morera y maíz (las mezclas 100:0 y 0:100), mostraron una pérdida en el volumen de 10 a $12 \%$, mientras que las mezclas intermedias (75:0, $50: 50$ y $25: 75)$ fueron de 4,6 a 5,5\%. Ambas observaciones indican que las mezclas de materiales con diferente densidad favorecen la compactación y acumulación de forraje fresco en un mismo volumen. 
Cuadro 3. Cantidad de materia seca y de nutrientes por tonelada de material ensilado al inicio y al final del experimento. Ochomogo, Cartago, Costa Rica. 2001.

\begin{tabular}{|c|c|c|c|c|c|}
\hline & \multicolumn{5}{|c|}{ Proporción de morera:maíz en el material ensilado } \\
\hline & 100:0\% & $75: 25 \%$ & $\mathbf{5 0 : 5 0 \%}$ & $25: 75 \%$ & $0: 100 \%$ \\
\hline \multicolumn{6}{|l|}{ Materia seca (kg/t MV) } \\
\hline Inicial & $224,40 \mathrm{a}$ & $221,00 \mathrm{~b}$ & $217,50 \mathrm{c}$ & $214,00 \mathrm{~d}$ & $210,40 \mathrm{e}$ \\
\hline Final & $192,80 \mathrm{a}$ & $191,60 \mathrm{a}$ & $190,30 \mathrm{a}$ & $189,50 \mathrm{a}$ & $177,20 \mathrm{~b}$ \\
\hline$\%$ pérdida & 14,08 & 13,30 & 12,51 & 11,45 & 15,78 \\
\hline \multicolumn{6}{|l|}{ Proteina cruda (kg/t MV) } \\
\hline Inicial & $36,24 \mathrm{a}$ & $31,09 \mathrm{~b}$ & $26,01 \mathrm{c}$ & $21,04 \mathrm{~d}$ & $16,16 \mathrm{e}$ \\
\hline Final & $26,82 \mathrm{a}$ & $26,56 \mathrm{a}$ & $24,45 \mathrm{a}$ & $19,92 \mathrm{~b}$ & $14,90 \mathrm{c}$ \\
\hline$\%$ pérdida & 26,00 & 14,60 & 5,99 & 5,32 & 7,77 \\
\hline$\%$ MS perdida & 4,20 & 2,05 & 0,72 & 0,52 & 0,60 \\
\hline \multicolumn{6}{|l|}{ FND (kg/t MV) } \\
\hline Inicial & $111,53 \mathrm{e}$ & $119,85 \mathrm{~d}$ & $127,91 \mathrm{c}$ & $135,76 \mathrm{~b}$ & $143,32 \mathrm{a}$ \\
\hline Final & $97,11 \mathrm{~b}$ & $97,79 \mathrm{~b}$ & $102,27 \mathrm{~b}$ & $115,29 \mathrm{a}$ & $114,63 \mathrm{a}$ \\
\hline$\%$ pérdida & 12,92 & 18,40 & 20,05 & 15,08 & 20,02 \\
\hline$\%$ MS perdida & 6,42 & 9,98 & 11,79 & 9,57 & 13,64 \\
\hline \multicolumn{6}{|l|}{ FAD $(\mathrm{kg} / \mathrm{t} \mathrm{MV})$} \\
\hline Inicial & $73,87 \mathrm{e}$ & $78,28 \mathrm{~d}$ & $82,52 \mathrm{c}$ & $86,65 \mathrm{~b}$ & $90,62 \mathrm{a}$ \\
\hline Final & $70,33 \mathrm{c}$ & $75,47 \mathrm{bc}$ & $76,71 \mathrm{ab}$ & $79,38 \mathrm{a}$ & $75,33 \mathrm{a}$ \\
\hline$\%$ pérdida & 4,79 & 3,59 & 7,04 & 8,39 & 16,87 \\
\hline$\%$ MS perdida & 1,58 & 1,27 & 2,67 & 3,40 & 7,27 \\
\hline \multicolumn{6}{|l|}{ Hemicelulosa (kg/t MV) } \\
\hline Inicial & $37,65 \mathrm{e}$ & $41,57 \mathrm{~d}$ & $45,39 \mathrm{c}$ & $49,11 \mathrm{~b}$ & $52,71 \mathrm{a}$ \\
\hline Final & $26,78 \mathrm{~b}$ & $22,32 \mathrm{~b}$ & $25,56 \mathrm{~b}$ & $35,91 \mathrm{a}$ & $39,30 \mathrm{a}$ \\
\hline$\%$ pérdida & 28,88 & 46,30 & 43,70 & 26,88 & 25,43 \\
\hline$\%$ MS perdida & 1,09 & 1,92 & 1,98 & 1,32 & 1,34 \\
\hline \multicolumn{6}{|l|}{ Lignina (kg/t MV) } \\
\hline Inicial & $14,03 \mathrm{e}$ & $13,88 \mathrm{~d}$ & $13,70 \mathrm{c}$ & $13,55 \mathrm{~b}$ & $13,38 \mathrm{a}$ \\
\hline Final & $13,21 \mathrm{a}$ & $13,49 \mathrm{a}$ & $13,49 \mathrm{a}$ & $12,58 \mathrm{a}$ & $10,30 \mathrm{~b}$ \\
\hline$\%$ pérdida & 5,83 & 2,81 & 1,53 & 7,11 & 23,06 \\
\hline$\%$ MS perdida & 0,36 & 0,18 & 0,10 & 0,45 & 1,47 \\
\hline \multicolumn{6}{|l|}{ Celulosa (kg/t MV) } \\
\hline Inicial & $59,85 \mathrm{e}$ & $64,40 \mathrm{~d}$ & $68,82 \mathrm{c}$ & $73,10 \mathrm{~b}$ & $77,24 \mathrm{a}$ \\
\hline Final & $57,13 \mathrm{~b}$ & $61,98 \mathrm{~b}$ & $63,22 b$ & $66,80 \mathrm{a}$ & $65,03 \mathrm{a}$ \\
\hline$\%$ pérdida & 3,68 & 3,09 & 6,79 & 7,27 & 13,47 \\
\hline$\%$ MS perdida & 0,27 & 0,24 & 0,56 & 0,63 & 1,22 \\
\hline \multicolumn{6}{|l|}{ Cenizas (kg/t MV) } \\
\hline Inicial & $33,55 \mathrm{a}$ & $28,80 \mathrm{~b}$ & $24,10 \mathrm{c}$ & $22,26 \mathrm{~d}$ & $19,15 \mathrm{e}$ \\
\hline Final & $27,84 \mathrm{a}$ & $26,29 \mathrm{a}$ & $23,35 \mathrm{~b}$ & $20,64 \mathrm{~b}$ & $15,58 \mathrm{c}$ \\
\hline$\%$ pérdida & 17,01 & 8,71 & 3,11 & 7,28 & 18,65 \\
\hline$\%$ MS perdida & 2,54 & 1,14 & 0,34 & 0,76 & 1,70 \\
\hline
\end{tabular}

a, b, c, d, e marca las diferencias significativas $(\mathrm{P}<0,05)$ entre tratamientos.

En las Figuras 1 y 2 se muestra la evolución de los contenidos de materia seca, proteína cruda, la fibra neutro detergente y sus partes constituyentes, entre las diferentes mezclas experimentadas. La concentración media de materia seca en la morera y en el maíz frescos fue entre 21 y $22,4 \%$, valores muy por debajo a los con- tenidos recomendados en el maíz (Johnson et al. 1999) y similares en la morera (González 1996) para obtener un ensilado de buena calidad. Bajo estas condiciones el ensilado mostró una pérdida en la concentración de materia seca cercano al $3 \%$, en forma constante en todas las mezclas. Las diferencias en la concentración de 


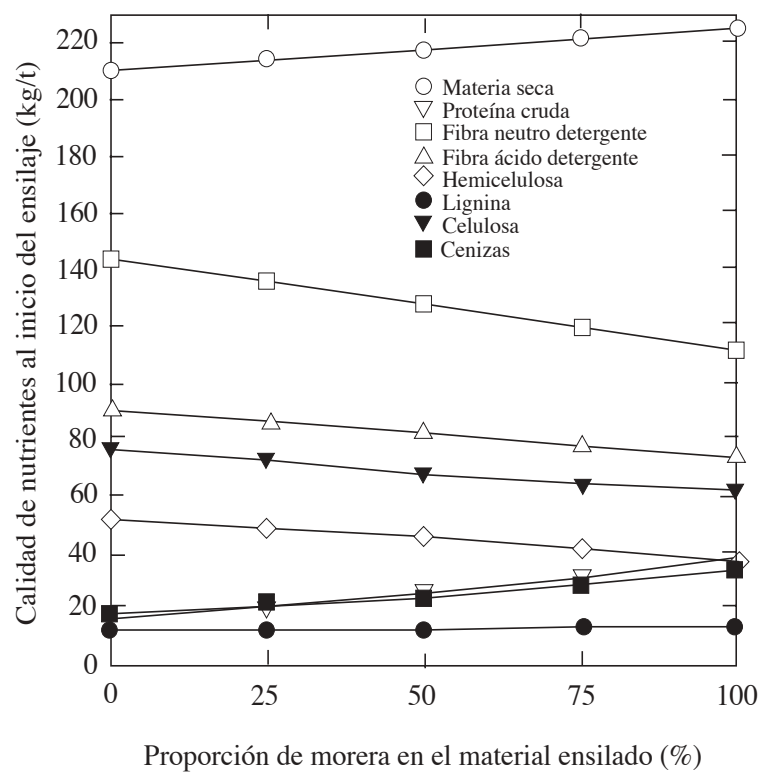

Figura 1. Composición química inicial de las mezclas estudiadas. Ochomogo, Cartago, Costa Rica. 2001.

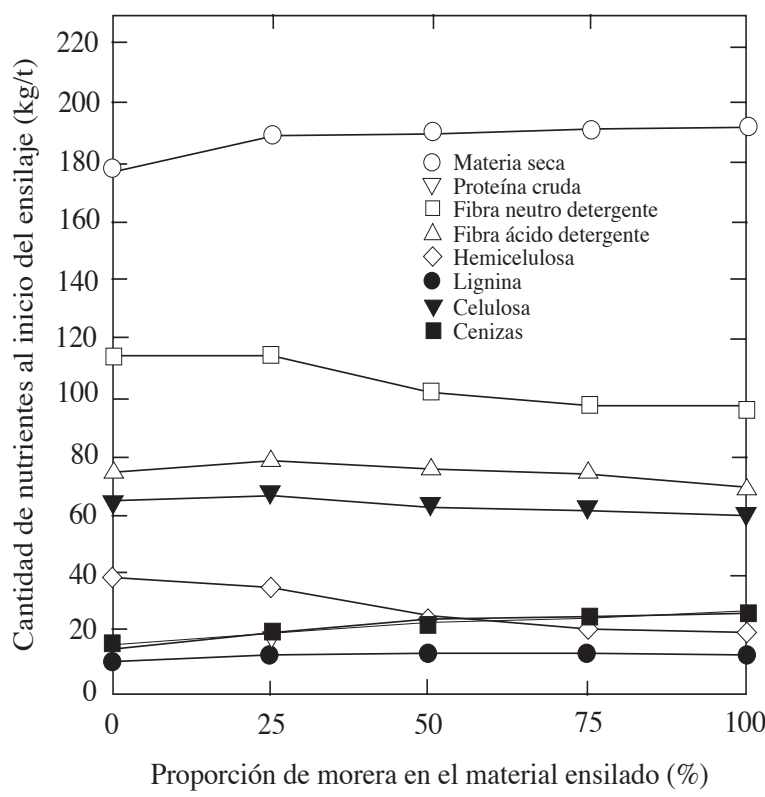

Figura 2. Composición química final de las mezclas estudiadas. Ochomogo, Cartago, Costa Rica. 2001.

proteína cruda y la fibra neutro detergente antes y después del proceso dentro de cada mezcla, se encuentran en similar magnitud, con valores similares a lo observado por González (1996; Ojeda et al. 2000), lo cual evidencia un proceso de fermentación uniforme y una con- servación de nutrientes apropiada y aceptable, a pesar de las grandes diferencias en la concentración de proteína, fibra y cenizas entre las mezclas.

\section{CONCLUSIONES Y RECOMENDACIONES}

Los resultados muestran que el ensilaje de morera, en mezcla pura (100:0), sufre un normal proceso de ensilaje, a pesar del alto contenido de proteína. En mezcla con maíz 75:25, 50:,50 y 25,75), se reduce a la mitad las pérdidas volumétricas, se aumenta la densidad del material almacenado por una mayor facilidad de compactación comparado con el ensilaje puro de maíz $(0: 100)$. Al observar los productos finales de la conservación de forrajes, todas las mezclas experimentadas mostraron un buen proceso de fermentación. Desde el punto de vista nutricional, se recomienda la adición de al menos un $25 \%$ de morera en la mezcla, observándose en la mezcla 50:50 un mejoramiento de $4-5 \%$ en la concentración de proteína cruda y disminución de $11 \%$ en la concentración de fibra neutro detergente sobre el ensilado puro de maíz. Bajo condiciones de excepción se recomienda el ensilaje de morera en la proporción 100:0, tomando en cuenta que el ensilado tendrá un ligero olor amoniacal.

\section{LITERATURA CITADA}

A.O.A.C. 1980. Methods of analysis. Ed. 13. Washington D.C. EUA. Association of Official Analysis Chemistry.

AMADOR, A. L; BOSCHINI, C. 2000. Fenología productiva y nutricional de maíz para la producción de forraje. Agronomía Mesoamericana 11:171-177.

BENAVIDES, J., LACHAUX, M. Y FUENTES, M. 1994. Efecto de la aplicación de estiércol de cabra en el suelo sobre la calidad y producción de biomasa de morera (Morus sp). Árboles y arbustos forrajeros en América Central. Serie Técnica. CATIE. Informe Técnico No 236. Volumen II. p. 495-514.

BOSCHINI, C; DORMOND, H; CASTRO, A. 1998. Producción de biomasa de morera (Morus alba) en la Meseta Central de Costa Rica. Agronomía Mesoamericana 9:31-40.

BOSCHINI, C; DORMOND, H; CASTRO, A. 1999. Composición química de la morera (Morus alba), para uso en la alimentación animal: densidades y frecuencias de poda. Agronomía Mesoamericana 11:41-49. 
BOSCHINI, C. 2001. Degradabilidad in situ de la materia seca, proteína cruda y fibra neutro detergente del forraje de morera (Morus alba). Agronomía Mesoamericana 12:79-87.

CASTRO, A. 1989. Producción de leche de cabras alimentadas con King Grass (Pennisetum purpureum x P. typloides), suplementales con diferentes niveles de follaje de Poró (E. poeppigrama) y de fruto de plátano (Musa sp. var. Pelipita). Tesis M.Sc. Turrialba, C.R. UCR/CATIE. $58 \mathrm{p}$.

ELIZONDO, J; BOSCHINI, C. 2002. Producción de forraje con maíz criollo y maíz híbrido. Agronomía Mesoamericana $13: 13-17$.

ESWARA, P; REDDY, M.R. 1992. Nutritive value of mulberry (Morus alba) leaves in goats and sheep. Indian Journal of Animal Science 8:295-296.

GOERING, H.K., VAN SOEST, P.J. 1970. Forage fiber analysis (Apparatus, reagents, procedures and some applications). Agricultural Handbook No. 379. ARS-USDA, Washington, DC.

GONZALEZ, J.G. 1996. Evaluación de la calidad nutricional de la morera fresca y ensilada con bovinos de engorde. Tesis de maestría. CATIE - Turrialba, Costa Rica. 58 p.

HUNT, C.W; KEZAR, W; HINMAN, D.D; COMBS, J.J; LOESCHE, J.A; MOEN, T. 1993. Effects of hybrid and ensiling with and without a microbial inoculant on the nutritional characteristics of whole plant corn. Journal of Animal Science 71:38-43.

HUNT, C.W; KEZAR, W; VINANDE, R. 1992. Yield, chemical composition, and ruminal fermentability of corn whole plant, ear, and stover as affected by hybrid. Journal of Production Agriculture 5:286-290.
JOHNSON, L; HARRISON, J.H; HUNT, C; SHINNERS, K; DOGGETT, C.G; SAPIENZA, D. 1999. Nutritive value of corn silage as affected by maturity and mechanical processing: a contemporary review. Journal of Dairy Science 82:2813-2825.

KUEHN, C.S; LINN, J.G; JOHNSON, D.G; JUNG, H.G; ENDRES, M.I. 1999. Effect of feeding silages from corn hybrids selected for leafness or grain to lactating dairy cattle. Journal of Dairy Science 82:2746-2755.

LEASK, W. C; DAYNARD, T.B. 1973. Dry matter yield, in vitro digestibility, percent protein, and moisture of corn stover following grain maturity. Canadian Journal of Plant Science 53:515-522.

OJEDA, F; MONTEJO, I; PÉREZ, G. 2000. Conservation of mulberry as silage. I. Effect on nitrogenous compounds. http://www.fao.org/ag/aga/agap/frg/afris/espanol/document/Irrd/Irrd01/2/benav102.htm. p 8.

ORTIZ, G. 1992. Efecto de la alimentación con pasto King Grass (Pennisetum purpureum $\mathrm{x}$ P. typohoides), suplementado con diferentes niveles de follaje de Morera (Morus alba) y de banano verde (Musa sp) sobre la producción de leche de cabra. Tesis Licenciatura. Escuela de Zootecnia. Universidad de Costa Rica. 45 p.

ROTH, G.W. 1994. Hybrid quality and yield differences for corn silage in Pennsylvania. Journal of Production Agriculture 7:50-54.

SAS. 1985. Statistical Analysis System. SAS User's Guide: Statistics (Version 5 Ed.) SAS Institute Inc. Cary, NC.

VERBIC, J; STEKAR, J. M. A; RESNIK-CEPON, M. 1995. Rumen degradation characteristics and fiber composition of various morphological parts of different maize hybrids and possible consequences for breeding. Animal Feeding Sciences Technology 54:133-148. 